\title{
NOVEL FEATURES OF MULTIPLICITY DISTRIBUTIONS IN QCD AND EXPERIMENT
}

\section{I.M. DREMIN}

\author{
Lebedev Physical Institute, Moscow 117924, Russia \\ CONTENTS
}

1. Introduction

2. Oscillations of cumulants of multiplicity distributions in QCD

3. Evolution of distributions with decreasing phase space volume intermittency and fractality

4. Zeros of truncated generating functions

5. Discussion and conclusions

\begin{abstract}
The solution of QCD equations for generating functions of multiplicity distributions reveals new peculiar features of cumulant moments oscillating as functions of their rank. This prediction is supported by experimental data on $e^{+} e^{-}, h h, A A$ collisions. Evolution of the moments at smaller phase space bins leads to intermittency and fractality. The experimentally defined truncated generating functions possess zeros in the complex plane of an auxiliary variable recalling Lee-Yang zeros in statistical mechanics.
\end{abstract}

\section{Introduction}

For a long time, the phenomenological approach dominated in description of multiplicity distributions in multiparticle production. The very first attempts to apply QCD formalism to the problem failed because in the simplest double-logarithmic approximation it predicts an extremely wide shape of the distribution that contradicts to experimental data. Only recently it became possible to get exact solutions of QCD equations which revealed much narrower shapes and such a novel feature of cumulant moments as their oscillations at higher ranks. These moments are extremely sensitive to the tiny details of the distribution. Surprisingly enough, those QCD predictions for parton distributions have been supported by experimental data for hadrons. 
QCD is also successful in qualitative description of evolution of these distributions with decreasing phase space bins which gives rise to notions of intermittency and fractality. However, there are some new problems with locations of the minimum of cumulants at small bins.

The experimentally defined truncated generating functions possess an intriguing pattern of zeros in the complex plane of an auxiliary variable. It recalls the pattern of Lee-Yang zeros of the grand canonical partition function in the complex fugacity plane related to phase transition.

Before demonstrating all these peculiarities let us define the multiplicity distribution

$$
P_{n}=\sigma_{n} / \sum_{n=0}^{\infty} \sigma_{n},
$$

where $\sigma_{n}$ is the cross section of $n$-particle production processes, and the generating function

$$
G(z)=\sum_{n=0}^{\infty} P_{n}(1+z)^{n}
$$

The (normalized) factorial and cumulant moments of the $P_{n}$ distribution are

$$
\begin{gathered}
F_{q}=\frac{\sum_{n} P_{n} n(n-1) \ldots(n-q+1)}{\left(\sum_{n} P_{n} n\right)^{q}}=\left.\frac{1}{\langle n\rangle^{q}} \frac{d^{q} G(z)}{d z^{q}}\right|_{z=0}, \\
K_{q}=\left.\frac{1}{\langle n\rangle^{q}} \frac{d^{q} \ln G(z)}{d z^{q}}\right|_{z=0},
\end{gathered}
$$

where $\langle n\rangle=\sum_{n} P_{n} n$ is the average multiplicity. They describe full and genuine $q$-particle correlations, correspondingly. Let us point out here that the moments are defined by the derivatives at the origin and are very sensitive to any nearby singularity of the generating function.

In practice, one deals with distribution truncated due to finiteness of the available phase space and the summation in all formulae above is cut off at some finite value of $n=N_{\max }$ which depends on the phase space region chosen, and increases with its increase. It is a polynomial of the power $N_{\max }$ and has $N_{\max }$ zeros in the complex $z$-plane.

To shorten the presentation, I omit here all the details of calculations and references to original papers. The reader can find them in my review paper in Physics-Uspekhi 37 (1994) 715. Main qualitative results are described and demonstrated in Figures in the subsequent three Sections. Their physics implications are discussed in the last Section. 


\section{Oscillations of cumulants of multiplicity dis- tributions in QCD}

First, let us consider QCD without quarks, i.e. gluodynamics. The generating function of the gluon multiplicity distribution in the full phase-space volume satisfies the equation

$$
\frac{\partial G(z, Y)}{\partial Y}=\int_{0}^{1} d x K(x) \gamma_{0}^{2}[G(z, Y+\ln x) G(z, Y+\ln (1-x))-G(z, Y)] .
$$

Here $Y=\ln \left(p \theta / Q_{0}\right), p$ is the initial momentum, $\theta$ is the angular width of the gluon jet considered, $p \theta \equiv Q$ where $Q$ is the jet virtuality, $Q_{0}=$ const,

$$
\gamma_{0}^{2}=\frac{6 \alpha_{S}(Q)}{\pi}
$$

$\alpha_{S}$ is the running coupling constant, and the kernel of the equation is

$$
K(x)=\frac{1}{x}-(1-x)[2-x(1-x)] .
$$

It is the non-linear integro-differential equation with shifted arguments in the non-linear part which take into account the conservation laws, and with the initial condition

$$
G(z, Y=0)=1+z
$$

and the normalization

$$
G(z=0, Y)=1 .
$$

The condition (9) normalizes the total probability to 1 , and the condition (8) declares that there is a single particle at the very initial stage.

After Taylor series expansion at large enough $Y$ and differentiation in eq. (5), one gets the differential equation

$$
(\ln G(Y))^{\prime \prime}=\gamma_{0}^{2}\left[G(Y)-1-2 h_{1} G^{\prime}(Y)+h_{2} G^{\prime \prime}(Y)\right],
$$

where $h_{1}=11 / 24 ; h_{2}=\left(67-6 \pi^{2}\right) / 36 \approx 0.216$, and higher order terms have been omitted.

Leaving two terms on the right-hand side, one gets the well-known equation of the double-logarithmic approximation which takes into account the 
most singular components. The next term, with $h_{1}$, corresponds to the modified leading-logarithm approximation, and the term with $h_{2}$ deals with nextto-leading corrections.

The straightforward solution of this equation looks very problematic. However, it is very simple for the moments of the distribution because $G(z)$ and $\ln G(z)$ are the generating functions of $F_{q}$ and $K_{q}$, correspondingly, according to (3), (4). Using this fact, one gets the solution which looks like

$$
H_{q}=\frac{K_{q}}{F_{q}}=\frac{\gamma_{0}^{2}\left[1-2 h_{1} q \gamma+h_{2}\left(q^{2} \gamma^{2}+q \gamma^{\prime}\right)\right]}{q^{2} \gamma^{2}+q \gamma^{\prime}},
$$

where the anomalous dimension $\gamma$ is related to $\gamma_{0}$ by

$$
\gamma=\gamma_{0}-\frac{1}{2} h_{1} \gamma_{0}^{2}+\frac{1}{8}\left(4 h_{2}-h_{1}^{2}\right) \gamma_{0}^{3}+O\left(\gamma_{0}^{4}\right) .
$$

The formula (11) shows how the ratio $H_{q}$ behaves in different approximations. In double-log approximation when $h_{1}=h_{2}=0$, it monotonously decreases as $q^{-2}$ that corresponds to the negative binomial law with its parameter $k=2$ i.e. to very wide distribution. In modified-log approximation $\left(h_{2}=0\right)$ it acquires a negative minimum at

$$
q_{\min }=\frac{1}{h_{1} \gamma_{0}}+\frac{1}{2}+O\left(\gamma_{0}\right) \approx 5
$$

and approaches asymptotically at large ranks $q$ the abscissa axis from below. In the next approximation given by (11) it preserves the minimum location but approaches a positive constant crossing the abscissa axis. In ever higher orders it reveals the quasi-oscillatory behavior about this axis. This prediction of the minimum at $q \approx 5$ and subsequent specific oscillations is the main theoretical outcome.

It is interesting to note that the equation (5) can be solved exactly in the case of fixed coupling constant. All the above qualitative features are noticeable here as well.

While the above results are valid for gluon distributions in gluon jets (and pertain to QCD with quarks taken into account), the similar qualitative features characterize the multiplicity distributions of hadrons in high energy reactions initiated by various particles. As an example, I show in Fig.1 the ratio $H_{q}$ as a function of $q$ in the $e^{+} e^{-}$data of DELPHI collaboration at $91 \mathrm{GeV}$, where the oscillations and the location of minima are of a special interest. 


\section{Evolution of distributions with decreasing phase-space volume - intermittency and frac- tality}

The multiplicity distributions can be measured not only in the total phase space (as has been discussed above for very large phase-space volumes) but in any part of it. For the homogeneous distribution of particles within the volume, the average multiplicity is proportional to the volume and decreases for small volumes but the fluctuations increase. The most interesting problem here is the law governing the growth of fluctuations and its possible departure from a purely statistical behavior related to the decrease of the average multiplicity. Such a variation has to be connected with the dynamics of the interactions. In particular, it has been proposed to look for the power-law behavior of the factorial moments for small rapidity intervals $\delta y$

$$
F_{q} \propto(\delta y)^{-\phi(q)} \quad(\phi(q)>0) \quad(\delta y \rightarrow 0),
$$

inspired by the idea of intermittency in turbulence. In the case of statistical fluctuations with purely Poissonian behavior, the intermittency indices $\phi(q)$ are identically equal to zero.

Experimental data on various processes in a wide energy range support this idea (e.g., see Fig.2), and QCD provides a good basis for its explanation as a result of parton showers. The generating function technique is not applicable here, and one should consider the Feynman graphs of evolution of a jet with its subjet hitting the phase-space window under consideration (see the abovementioned review).

At moderately small rapidity windows, one can get in the double-log approximation the power-law behavior with

$$
\phi(q)=D(q-1)-\frac{q^{2}-1}{q} \gamma_{0} .
$$

The running property of QCD coupling constant is not important in that region. This property becomes noticeable at ever smaller windows when (e.g., at $q=2) \ln \delta y_{0} / \delta y>\alpha_{S}^{-1}$, and leads to smaller numerical values of $\phi(q)$ compared to (15). The general trends in this region decline somewhat from the

simple power law (14) due to logarithmic corrections. Qualitatively, these 
predictions correspond to experimental findings at relatively small ranks $q$ where the steep increase in the region of $\delta y>1$ on the log-log plot of the dependence (14) is replaced by slower one at smaller intervals $\delta y$ (see Fig.2). The transition point between the two regimes depends on the rank in qualitative agreement with QCD predictions also. Namely, the transition happens at smaller bins for higher ranks. These findings can be interpreted as an indication on fractal structure of particle distributions within the available phase space. When interpreted in terms of fluctuations, they show that the fluctuations become stronger in small phase-space regions in a definite power-like manner and, surely, exceed trivial statistical fluctuations.

Let us turn now to the $q$-behavior of moments at small bins. The phenomenon of the oscillations of cumulants discussed above reveals itself here as well if one goes beyond the double-log approximation of (15). In terms of factorial moments, it means the non-monotonous behavior of the intermittency indices as functions of $q$. (Compare it to the steady increase with $q$ at $q>1$ given by (15).) It gives rise to the negative values of $K_{q}$ and $H_{q}$.

The fate of the first minimum can be easily guessed from the formula (13). For large enough virtualities (i.e. small $\gamma_{0}$ ), the minimum location moves to higher values of rank $q$ for jets with larger virtuality $Q$ since the QCD coupling constant is running as $\ln ^{-1} Q$. Therefore, the predicted shift of the minimum is

$$
q_{\min } \propto \ln ^{1 / 2} Q
$$

It follows that $q_{\min }$ moves to higher ranks at higher energies because more massive jets become available. Another corollary is that it should shift to smaller values of $q$ for smaller bins at fixed energy.

While former statement finds some support in experiment, the second one does not look to be true as shown in Fig.3. On the contrary, the minimum appears at higher ranks for smaller bins. There is no solution of this problem yet but it should be ascribed to the higher-order effects. Actually, one can guess that the higher order terms shown as $O\left(\gamma_{0}\right)$ in (13) become so important at small bins that they overpower the weak $Q$-dependence of $\gamma_{0}^{-1}$ in the first term of (13). It is important to stress here that at large rapidity intervals the modified leading-log term with $h_{2}$ does not influence the value of $q_{\text {min }}$, and only increases the value of $H_{q}$ by $h_{2} \gamma_{0}^{2}$. Thus, the next- to-leading corrections should be in charge of the additional shift of $q_{m i n}$, and, therefore, small bins help us look into higher orders of QCD. 


\section{Zeros of truncated generating functions}

There is another fascinating feature of multiplicity distributions - it happens that zeros of the truncated generating function form a spectacular pattern in the complex plane of the variable $z$. Namely, they seem to lie close to a single circle. At enlarged values of $N_{\max }$ they move closer to the real axis pinching it at some positive value of $z$. It is demonstrated in Fig.4 for UA5 data on $p \bar{p}$ interactions at 200 and $900 \mathrm{GeV}$ for various rapidity windows.

No QCD interpretation of the fact exists because it is hard to exploit the finite cut-off in analytic calculations. The interest to it stems from the analogy to the locations of zeros of the grand canonical partition function as described by Lee and Yang who related them to possible phase transitions in statistical mechanics. In that case, $z$ variable plays the role of fugacity, and pinching of the real axis implies existence of two phases in the system considered.

In particle physics, it shows up the location of the singularity of the generating function i.e. the number of zeros of truncated generating functions increases and they tend to move to the singularity point when $N_{\max } \rightarrow$ $\infty$. Since it happens to lie close to the origin, it drastically influences the behavior of moments (see (3), (40), and, therefore, determines the shape of the distribution. The study of the singularities is at the very early stage now, and one can only say that the singularity is positioned closer to the origin in nucleus-nucleus collisions and it is farthest in $e^{+} e^{-}$that appeals to our intuitive guess.

\section{Discussion and conclusions}

Let us discuss the implications of the above findings. The QCD prediction of quasioscillating behavior of cumulant moments of the multiplicity distributions reveals the tiny features which were overlooked by simpleminded fits of the negative binomial distribution. The truncated negative binomial distribution has the oscillating cumulants as well but the oscillations should die out asymptotically while they persist in QCD.

It demonstrates that the QCD distribution belongs to the class of noninfinitely-divisible ones and shows that the Poissonian cluster models are ruled out by QCD since they lead to positive values of cumulants and can 
not reproduce their oscillations at asymptotically high energies.

Moreover, this prediction is closely related to the existence of a new expansion parameter in describing multiparticle production in QCD. In terms of moments, this parameter is equal to the product of the moment's rank to the anomalous dimension of QCD $\gamma q$. Let us recall that the similar parameter appears when calculating the Feynman tree graphs and looks like a product of the number of final particles to QCD coupling constant $n \alpha_{S}$. Both parameters become large if large number of particles is involved.

The invalidity of simple perturbative approach in multiple production processes would ask for more convenient basis (compared to particle number representation) to be used. As one of possible examples of effectiveness of such an approach, we would mention lasers where coherent state basis is more suitable. That is why studies of coherent and squeezed states as well as statistical approach and search for collective effects, in general, are welcome. The recent findings of zeros of the truncated generating functions point out in that direction, and provoke speculations to their possible relation to the problem of phase transition in hadronic matter.

The success of QCD in predicting the qualitative features of moments of multiplicity distributions both for large phase-space regions (oscillation of cumulants) and for small bins (intermittency indices, fractality) seems even more surprising if one recognizes that they are derived for parton distributions while hadrons are observed in experiment. Nevertheless, these qualitative features reveal themselves in hadron distributions as well. Why such a local parton-hadron duality persists even at higher moments is an open question. Another problem appears in such a tiny property as the evolution of the minimum location at smaller bins. Its solution can show a way to take into account higher order effects of QCD more properly.

To conclude, we would like to stress that recent theoretical studies of QCD multiplicity distributions predict rather exotic features of the moments of the distributions which find some support in experiment and, at the same time, provoke new problems to be solved.

\section{Acknowledgements}

I am grateful to Takeshi Kodama for inviting me to participate in this very fruitful workshop and for financial support.

This work is supported by the Russian Fund for Fundamental Research (grant 93-02-3815), by Soros Foundation (grant M5V300) and by INTAS- 
930-0079 grant.

\section{FIGURE CAPTIONS}

Fig.1 The ratio of cumulant to factorial moments $H_{q}$ as the function of the rank $q$. The DELPHI-data on $e^{+} e^{-}$at $91 \mathrm{GeV}$ in the total phase space are shown by dots. The dashed line shows the fit by the negative binomial distribution with the parameters given by DELPHI. The solid line is drawn just to guide the eye.

Fig. 2 Factorial moments of order $q=2, \ldots, 4$ for the all-charged, positivesonly and negatives-only samples of NA22-data on $\pi^{+} p$ interactions at 250 $\mathrm{GeV} / \mathrm{c}$ (lab. system).

Fig.3 Behavior of the ratio of cumulant to factorial moments $H_{q}$ as a function of the rank $q$ for some single hemisphere multiplicity distributions from the DELPHI collaboration: (a) full hemisphere; (b) rapidity window $|y| \leq 1.5$; (c) rapidity window $|y| \leq 1.0$. The minimum shifts to higher $q$ for smaller bins.

Fig.4 The locations of zeros of the truncated generating function for UA5data on $p \bar{p}$ interactions at 200 and $900 \mathrm{GeV}$ (c.m.s.) in various rapidity windows. The upper halfplane of $z=x+i y$ is shown only because of the up-down symmetry. (For further experimental information see the talk of G. Gianini at Multiparticle Dynamics-95). 
This figure "fig1-1.png" is available in "png" format from: http://arxiv.org/ps/hep-ph/9509377v1 
This figure "fig1-2.png" is available in "png" format from: http://arxiv.org/ps/hep-ph/9509377v1 
This figure "fig1-3.png" is available in "png" format from: http://arxiv.org/ps/hep-ph/9509377v1 